\title{
Complementary medicine use in US adults with a history of colorectal cancer: a nationally representative survey
}

\author{
Charlene HL Wong ${ }^{1} \cdot$ Tobias Sundberg ${ }^{2,3} \cdot$ Vincent $\mathrm{CH}$ Chung ${ }^{1,3,4} \cdot$ Petra Voiss ${ }^{5} \cdot$ Holger Cramer $^{3,5}$ (1)
}

Received: 6 March 2020 / Accepted: 20 April 2020 / Published online: 1 May 2020

(C) The Author(s) 2020

\begin{abstract}
Background In the USA, colorectal cancer is among the top diagnosed cancers. The current study specifically targets the US adult population that have a history of colorectal cancer.

Methods We used the 2017 National Health Interview Survey (NHIS) to investigate the prevalence and predictors of colorectal cancer survivors using complementary medicine in the past 12 months in a representative sample of the US population $(N=$ 26,742). We descriptively analyzed the 12-month prevalence of any complementary medicine use separately for individuals with a prior diagnosis of colorectal cancer and those without. Using chi-squared tests and backward stepwise multiple logistic regression analyses, we identified predictors of complementary medicine use in the past 12 months.

Results A weighted total of $1,501,481$ US adults $(0.6 \%)$ had a history of colorectal cancer. More individuals without (weighted $n=76,550,503 ; 31.2 \%$ ) than those with a history of colorectal cancer (weighted $n=410,086 ; 27.3 \%$ ) had used complementary medicine. The most commonly used complementary medicine among colorectal cancer patients was mind-body medicine, followed by chiropractic. A higher prevalence of complementary medicine use was associated with being female, higher educated and/or living in the US Midwest or South.

Conclusions In this study, over one fourth of the US colorectal cancer survivors had used complementary medicine. Mind-body medicine was found to be the most commonly used. With evidence supporting the effectiveness and safety of mind-body medicine use among colorectal cancer patients, promoting the use of evidence-based mind-body medicine for colorectal cancer management could be considered.
\end{abstract}

Keywords Colorectal cancer $\cdot$ Complementary therapies $\cdot$ Mind-body medicine $\cdot$ Public health

Holger Cramer

h.cramer@kem-med.com

1 Jockey Club School of Public Health and Primary Care, The Chinese University of Hong Kong, Hong Kong, China

2 Musculoskeletal and Sports Injury Epidemiology Center (MUSIC), Institute of Environmental Medicine, Karolinska Institutet, Stockholm, Sweden

3 Australian Research Centre in Complementary and Integrative Medicine (ARCCIM), Faculty of Health, University of Technology Sydney, Sydney, New South Wales, Australia

4 School of Chinese Medicine, The Chinese University of Hong Kong, Hong Kong, China

5 Department of Internal and Integrative Medicine, Evang. Kliniken Essen-Mitte, Faculty of Medicine, University of Duisburg-Essen, Am Deimelsberg 34a, 45276 Essen, Germany

\section{Introduction}

The populations of North America, along with those in Europe, Australia, New Zealand, and Eastern Asia, have the highest age standardized incidence rates of colorectal cancer in the world [1]. In the USA, colorectal cancer is among the top diagnosed cancers [2], and in 2018, colorectal cancer represented $8.1 \%$ of all new cancer cases [3]. The lifetime risk of US men and women of developing colorectal cancer has been estimated to $4.2 \%$ [3]. It has been estimated that the global burden of colorectal cancer will grow by $60 \%$ over the next decade to encompass more than 2.2 million new cases and 1.1 million deaths by 2030 [4]. Considering the variation of the incidence of colorectal cancer between different regions and cultures, factors that may contribute to the expected increase are the economical and developmental changes in many lowand middle-income countries including the adoption of western lifestyle [4]. Similarly, it has been suggested that different 
lifestyle and dietary habits may be important factors for colorectal cancer prevalence $[5,6]$.

Current strategies and guidelines for colorectal diagnosis and treatment include screening and physician investigations to identify cancer pathology that if found can be treated both with local and systematic interventions such as surgery, chemotherapy, radiation therapy, and local ablation [7-10]. Of those being diagnosed with colorectal cancer in the USA, $64.5 \%$ are expected to survive 5 years or more after having received their diagnosis [3]. Albeit significant betterments in the treatment of patients with colorectal cancer and metastatic disease over the last decades [2], there are still significant burdens and suffering accompanying this disease. Living with colorectal cancer may relate to both physical and psychological health consequences including distress, depression, and bowel problems [11].

Living with colorectal cancer in the long-term typically involves continued conventional care and follow-ups to monitor cancer and medical health status. Recent research also supports the value of colorectal cancer patients to be physically active, which may lead to better quality of life [12]. It has also been reported that patients with colorectal cancer might complement their reliance of conventional care with other types of treatments and activities including the use of complementary medicines, whereby some research suggest that up to $75 \%$ of patients have used at least one complementary therapy [13]. Previous research targeting the US population report that $79 \%$ of cancer survivors use complementary medicine, and that those users were slightly more likely to be survivors of colorectal, breast, or melanoma cancers [14]. It was further reported that cancer patients' main reasons for using complementary medicine was to support general wellness and pain and for cardiovascular reasons rather than for cancer specific concerns [14].

The current study specifically targets the US adult population that have a history of colorectal cancer and investigates the prevalence and predictors of them using complementary medicine.

\section{Methods}

\section{Study design}

We analyzed data from the 2017 US National Health Interview Survey (NHIS), a nationally representative interview survey monitoring the health of the noninstitutionalized US population. More information on survey composition, sampling strategy, and administration of the NHIS can be found online [15]. A total of 32,617 households were included in the survey and 26,742 adults provided data (response rate: $80.7 \%$ ) [16].
Specifically, we used data from the NHIS Person File and NHIS Sample Adult File. Prior cancer diagnoses were queried as follows: "Have you ever been told by a doctor or other health professional that you had cancer or a malignancy of any kind?" If this question was answered in the affirmative, the participant was asked "What kind of cancer was it?" Up to three different kinds of cancer were collected. We considered all participants who indicated that they had been told to have had colon cancer and/or rectal cancer as having received a diagnosis of colorectal cancer. We further included data on time since diagnosis (operationalized as age at time of the survey minus age at first diagnosis) as well as the sociodemographic characteristics age, sex, ethnicity, region, marital status, education, and employment. We defined complementary medicine use as having consulted with chiropractors, naturopaths, practitioners of chelation therapy, practitioners of traditional medicine, and/or homeopaths in the past 12 months and/or having used mind-body medicine approaches in the past 12 months. We considered the use of mantra meditation, mindfulness meditation, spiritual meditation, guided imagery, progressive relaxation, yoga, tai chi, and/or qi gong as having used mind-body medicine.

\section{Statistical analysis}

We descriptively analyzed the 12-month prevalence of any complementary medicine use separately for individuals with a prior diagnosis of colorectal cancer and those without such a diagnosis. We further analyzed the 12-month prevalence of consultations chiropractors, naturopaths, practitioners of chelation therapy, practitioners of traditional medicine, homeopaths, and/or of the use mind-body medicine approaches. Since the NHIS oversamples minorities, we calculated population-based estimates using weights calibrated to the 2010 census-based population estimates for age, gender, and ethnicity of the US civilian non-institutionalized population.

Using chi-squared tests, we compared socio-demographic and clinical characteristics between (a) individuals with versus those without a prior diagnosis of colorectal cancer, and (b) individuals with a prior diagnosis of colorectal cancer who had used versus those who had not used complementary medicine in the past 12 months. We included the following independent variables in the analysis: age (categories: 18-29, 30 39, 40-49, 50-64, 65 years or older), ethnicity (categories: non-Hispanic White, Hispanic, African American, Asian, Other), region (categories: West, Northeast, Midwest, South), marital status (categories: not in relationship; in relationship), education (categories: less than college, some college, or more), employment (categories: employed, unemployed), and time since cancer diagnosis (categories: up to 1 year, $2-5$ years, more than 5 years).

To analyze independent predictors of complementary medicine use in the past 12 months, we utilized backward stepwise 
multiple logistic regression analyses. Including too many potential predictor variables in the analysis can dilute true associations due to wide confidence intervals or identify spurious associations [17]. We therefore only considered those potential predictors associated with mind-body medicine use in univariate analysis (chi-squared test) for the multivariate analysis. The cutoff for significance in univariate analysis was chosen more liberal ( $p$ value of $\leq 0.10$ ) than common since its purpose was to identify potential predictor variables rather than to test a hypothesis [17]. In the multivariate analysis, we calculated adjusted odds ratios with $95 \%$ confidence intervals $(\mathrm{CI})$ and $P$ values using relative weights and considered $P$ values of $\leq$ 0.05 statistically significant in regression analysis. We used the Statistical Package for Social Sciences (IBM SPSS Statistics for Windows, release 25.0. Armonk, NY: IBM Corp.) for all analyses.

\section{Results}

We found that a weighted total of $1,501,481$ US adults $(0.6 \%)$ had a history of colorectal cancer. More individuals without (weighted $n=76,550,503 ; 31.2 \%$ ) than those with a history of colorectal cancer (weighted $n=410,086 ; 27.3 \%$ ) had used complementary medicine. In detail, the 12-month prevalence of consultations with a chiropractor and of using mind-body medicine was higher in individuals without a diagnosis (Fig. 1). The prevalence of consultations with naturopaths, practitioners of traditional medicine was higher in those with a prior colorectal cancer diagnosis (Fig. 1). Besides complementary medicine use, individuals with and without a history of colorectal cancer also differed on several sociodemographic characteristics (Table 1).

In univariate analysis, individuals with a prior colorectal cancer diagnosis using complementary medicine were more likely female, higher educated and/or living in the US Midwest or South than those not using complementary medicine (Table 2). In regression analyses, we found that independent predictors of complementary medicine use in individuals with a prior diagnosis of colorectal cancer included the following: women had 4.15 times the odds (95\% CI 1.94 to 8.85; $p<0.001$ ) of using complementary medicine compared to men, and individuals with at least some college education had 2.78 times the odds (95\% CI 1.18 to $6.54 ; p=0.020$ ) compared with less educated individuals.

\section{Discussion}

\section{Summary of findings}

In this nationally representative interview survey, there was $0.6 \%$ of the US adults who reported a prior colorectal cancer diagnosis, and $27.3 \%$ of these cancer patients had used complementary medicine. The most commonly used complementary medicine among colorectal cancer patients was mindbody medicine, followed by chiropractic services. However, their prevalence was even higher in individuals without a diagnosis. While the prevalence of consultations with naturopaths, practitioners of traditional medicine was higher in those with a prior colorectal cancer diagnosis, these analyses were based on less than 10 individuals with a diagnosis and are such prone to bias.

Furthermore, being female, having higher education level and living in the US Midwest or South were associated with an increased consumption of complementary medicine. The predictors of their complementary medicine use were female gender and higher education level.

\section{Use of complementary medicine among colorectal cancer patients in other Western countries}

While our nationally representative interview survey indicated that $27.3 \%$ of colorectal cancer patients in the USA had used
Fig. 1 12-month prevalence of consultations with complementary medicine practitioners and of mind-body medicine use in individuals with and without a history of colorectal cancer. Weighted frequencies were used

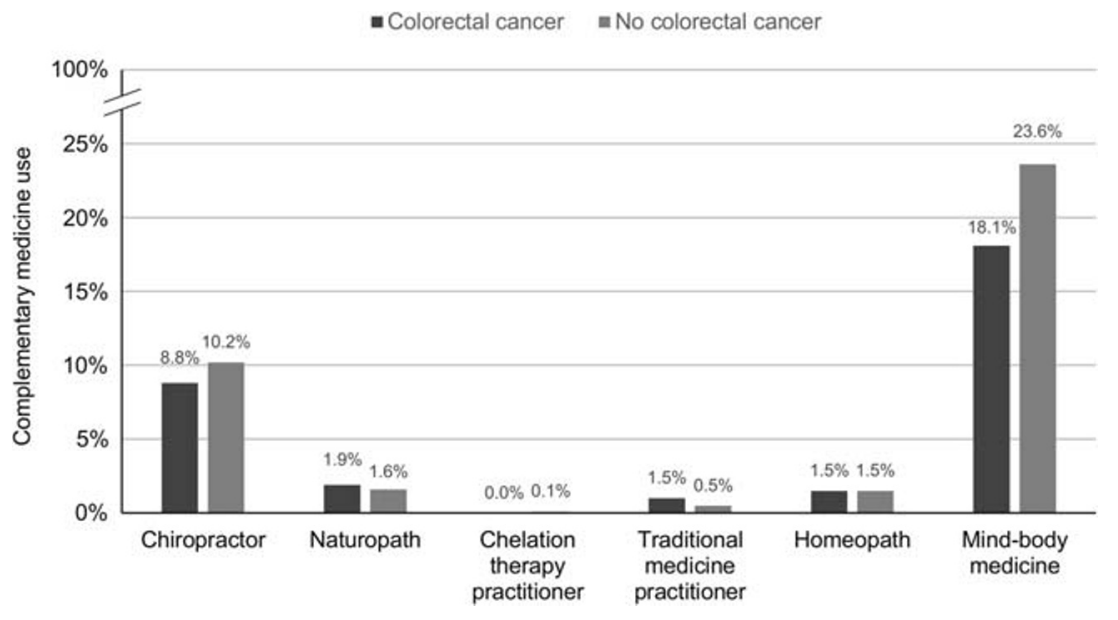


Table 1 Comparison of characteristics in individuals with and without a prior colorectal cancer diagnosis. Weighted frequencies are reported; $P$ values are derived from chi-squared tests using relative weights

\begin{tabular}{|c|c|c|c|}
\hline Characteristics & $\begin{array}{l}\text { Individuals without colorectal cancer } \\
\text { (weighted } n=245,155,790 \text { ) }\end{array}$ & $\begin{array}{l}\text { Individuals with colorectal cancer } \\
\text { (weighted } n=1,501,481 \text { ) }\end{array}$ & $P$ values \\
\hline Age & & & $<0.001$ \\
\hline 18 to 29 years & $51,911,205(21.1 \%)$ & $0(0.0 \%)$ & \\
\hline 30 to 39 years & $42,100,721(17.2 \%)$ & $113,879(7.6 \%)$ & \\
\hline 40 to 49 years & $39,632,186(16.2 \%)$ & $64,613(4.3 \%)$ & \\
\hline 50 to 64 years & $62,818,950(25.6 \%)$ & $488,732(32.5 \%)$ & \\
\hline 65 years and up & $48,692,728(19.9 \%)$ & $834,257(55.6 \%)$ & \\
\hline Sex & & & 0.052 \\
\hline Male & $118,152,291(48.2 \%)$ & $837,797(55.8 \%)$ & \\
\hline Female & $127,003,499(51.8 \%)$ & $663,684(44.2 \%)$ & \\
\hline Ethnicity & & & $<0.001$ \\
\hline Non-Hispanic White & $158,136,596(64.5 \%)$ & $1,192,554(79.4 \%)$ & \\
\hline Hispanic & $39,280,118(16.0 \%)$ & $135,625(9.0 \%)$ & \\
\hline Black & $30,113,985(12.3 \%)$ & $72,252(4.8 \%)$ & \\
\hline Asian & $14,840,605(6.1 \%)$ & $99,141(6.6 \%)$ & \\
\hline Other & $2,784,486(1.1 \%)$ & $1909(0.1 \%)$ & \\
\hline Region & & & $<0.001$ \\
\hline West & $58,127,572(23.7 \%)$ & $249,900(16.6 \%)$ & \\
\hline Northeast & $44,723,365(18.2 \%)$ & $445,695(29.7 \%)$ & \\
\hline Midwest & $53,423,558(21.8 \%)$ & $369,211(24.6 \%)$ & \\
\hline South & $88,881,295(36.3 \%)$ & $436,675(29.1 \%)$ & \\
\hline Employment & & & $<0.001$ \\
\hline Unemployed & $91,334,035(37.3 \%)$ & $935,471(62.3 \%)$ & \\
\hline Employed & $153,745,086(62.7 \%)$ & $566,010(37.7 \%)$ & \\
\hline Education & & & 0.405 \\
\hline Less than college & $88,094,863(35.9 \%)$ & $488,434(32.5 \%)$ & \\
\hline Some college or more & $156,076,430(63.7 \%)$ & $992,350(66.1 \%)$ & \\
\hline Marital status & & & $<0.001$ \\
\hline Not in a relationship & $97,396,032(39.7 \%)$ & $404,582(26.9 \%)$ & \\
\hline In a relationship & $147,382,833(60.1 \%)$ & $1,096,899(73.1 \%)$ & \\
\hline
\end{tabular}

complementary medicine, a former literature review showed that approximately $75 \%$ of colorectal cancer patients in Europe and Canada consumed at least one complementary medicine approach to improve their general health and physical well-being [13].

A Canadian survey demonstrated that patients' use of complementary medicine increased dramatically following their colorectal cancer diagnosis as they would like to improve their bodies' capacity to fight cancer [18]. Another survey indicated that the majority of colorectal cancer patients in Europe felt satisfied with the use of complementary medicine [19]. They believed that complementary medicine may bring them benefits in psychosocial functioning, positive effects in their empowerment, and direct involvement in their cancer care [20]. Other possible reasons for patients to use complementary medicine included patients' intention to try each available treatment option and less concerns on the safety of complementary medicine modalities than conventional treatments [21].

\section{Predictors of the use of complementary medicine among colorectal cancer patients}

Our findings were in line with previous studies on the complementary medicine use for managing cancer symptoms [22, 23], which showed that female gender and higher education level were predictors of using complementary medicine among colorectal cancer patients. These two predictors were also found to be associated with an increasing consumption of complementary medicine among patients in our study. 
Table 2 Comparison of characteristics in individuals with a prior colorectal cancer diagnosis using or not using complementary medicine. Weighted frequencies are reported; $P$ values are derived from chi-squared tests using relative weights

\begin{tabular}{|c|c|c|c|}
\hline Characteristics & $\begin{array}{l}\text { Not using complementary medicine } \\
\text { (weighted } n=1,091,395 \text { ) }\end{array}$ & $\begin{array}{l}\text { Using complementary medicine } \\
\text { (weighted } n=410,086 \text { ) }\end{array}$ & $P$ values \\
\hline Age & & & 0.159 \\
\hline 18 to 29 years & $0(0.0 \%)$ & $0(0.0 \%)$ & \\
\hline 30 to 39 years & $69,678(6.4 \%)$ & $4.4201(10.8 \%)$ & \\
\hline 40 to 49 years & $26,533(2.4 \%)$ & $38,080(9.3 \%)$ & \\
\hline 50 to 64 years & $345,996(31.7 \%)$ & $142,736(34.8 \%)$ & \\
\hline 65 years and up & $649,188(59.5 \%)$ & $185,069(45.1 \%)$ & \\
\hline Sex & & & $<0.001$ \\
\hline Male & $703,785(64.5 \%)$ & $134,012(32.7 \%)$ & \\
\hline Female & $387,610(35.5 \%)$ & $276,074(67.3 \%)$ & \\
\hline Ethnicity & & & 0.090 \\
\hline Non-Hispanic White & $867,363(79.5 \%)$ & $325,191(79.3 \%)$ & \\
\hline Hispanic & $68,115(6.2 \%)$ & $67,510(16.5 \%)$ & \\
\hline Black & $59,807(5.5 \%)$ & $12,445(3.0 \%)$ & \\
\hline Asian & $96,110(8.8 \%)$ & $3031(0.7 \%)$ & \\
\hline Other & $0(0.0 \%)$ & $1909(0.5 \%)$ & \\
\hline Region & & & 0.038 \\
\hline West & $160,401(14.7 \%)$ & $89,499(21.8 \%)$ & \\
\hline Northeast & $388,971(35.6 \%)$ & $56,724(13.8 \%)$ & \\
\hline Midwest & $235,449(21.6 \%)$ & $133,762(32.6 \%)$ & \\
\hline South & $306,574(28.1 \%)$ & $130,101(31.7 \%)$ & \\
\hline Education & & & 0.039 \\
\hline Less than college & $401,486(36.8 \%)$ & $869.48(21.2 \%)$ & \\
\hline Some college or more & $669,212(61.3 \%)$ & $323,138(78.8 \%)$ & \\
\hline Employment & & & 0.875 \\
\hline Unemployed & $685,925(62.8 \%)$ & $249,546(60.9 \%)$ & \\
\hline Employed & $405,470(37.2 \%)$ & $160,540(39.1 \%)$ & \\
\hline Marital status & & & 0.399 \\
\hline Not in a relationship & $274,922(25.2 \%)$ & $129,660(31.6 \%)$ & \\
\hline In a relationship & $816,473(74.8 \%)$ & $280,426(68.4 \%)$ & \\
\hline Years since diagnosis & & & 0.198 \\
\hline Up to 1 year & $255,552(23.4 \%)$ & $49,800(12.1 \%)$ & \\
\hline $2-5$ years & $271,318(24.9 \%)$ & $97,826(23.9 \%)$ & \\
\hline More than 5 years & $561,181(51.4 \%)$ & $262,460(64.0 \%)$ & \\
\hline
\end{tabular}

A certain number of psychological factors were found to be related to the use of complementary medicine among female colorectal cancer patients, namely, worrying of cancer recurrence, vigor, anger, and emotional distress [24]. As complementary medicine was suggested to be effective in improving psychosocial functioning [25], female patients may be more likely to consume it due to this potential benefit. Besides, it is noteworthy that cancer patients with higher education level may have an inherent negative attitude towards conventional medicine, as well as higher awareness of complementary medicine modalities [26]. These factors may account for colorectal cancer patients' choice for complementary medicine.

\section{Potential risk of non-disclosure of complementary medicine use to physicians}

Since the non-disclosure of complementary medicine use to physicians may possibly lead to drug interactions and adverse interactions with conventional pharmaceutical treatments [27, 28] among colorectal cancer patients, it is important to strengthen physicians' knowledge about complementary medicine and their communications with patients $[18,29]$. This may enhance the mutual trust between physicians and patients and increase their abilities on healthcare decision-making [30, $31]$. 
A survey in Denmark demonstrated that over half of the colorectal cancer patients failed to disclose their consumption of complementary medicine to their physicians, and less than $10 \%$ reported to have been asked by their physicians about the use of complementary medicine [32]. In addition, nondisclosure of complementary medicine use is also common among cancer patients in the USA. The 2012 US NHIS also indicated that about $30 \%$ of cancer patients who consumed complementary medicine did not disclose the use of complementary medicine to their physicians [33]. The most frequently reported reasons for their non-disclosure of complementary medicine use were (i) the physician did not ask and (ii) the patients did not think that their physicians needed to know [33].

In our survey, the colorectal cancer patients' disclosure of complementary medicine use to physicians was not assessed. Future rounds of NHIS may investigate the communications between physicians and colorectal cancer patients about the consumption of complementary medicine. The findings would inform the development of effective strategies to enhance patients' disclosure of complementary medicine use and reduce the risk of adverse effects among patients [29].

\section{Mind-body medicine as the most commonly used complementary medicine for managing colorectal cancer}

Findings of our study concur with those of the previous literature review [13] that mind-body medicine was one of the most commonly used form of complementary medicine modality among colorectal cancer patients. Since it is not uncommon for cancer patients to experience symptoms such as anxiety, pain, fatigue, and decreased quality of life, mind-body medicine which aims to use one's mind to improve physical function and enhance health are becoming popular among cancer patients [34].

Existing evidence supports the effectiveness of different mind-body medicine modalities among colorectal cancer patients. For instance, mindfulness meditation and progressive relaxation showed significant effects in relieving stress [35, 36 , while yoga and qigong are effective in reducing anxiety and improving sleep quality [37, 38]. Besides, qigong showed benefits in reducing fatigue, improving physical activity, and quality of life [39]. These mind-body modalities are safe in general, as long as they are practiced under the guidance from qualified instructors [40, 41].

Promoting rational use of evidence-based mind-body medicine in the local colorectal cancer community could therefore be considered. Future NHIS may investigate reasons and expenditure for mind-body medicine use among the US patients. This will identify patients' possible clinical and biopsychosocial needs, as well as costs on consuming mindbody medicine. The promotion strategies for mind-body medicine among colorectal cancer patients will then be facilitated accordingly.

\section{Strengths and limitations}

There are some strengths and limitations in this study. Since the 2017 NHIS focused on the US nationally representative sample of the population, it provided a robust epidemiological basis for investigating the patients' characteristics and predictors on the consumption of healthcare services. This study offers critical insight into the prevalence, patterns, and predictors of complementary medicine use among colorectal cancer patients. Moreover, our findings may inform research funders to allocate resources on various research projects related to complementary medicine use, particularly on mind-body medicine, among these cancer patients.

Nonetheless, this is a cross-sectional study which only shows the associations between the use of complementary medicine and patients' characteristics without examining the casual relationship. Our study is a secondary analysis of the existing data. Reasons for the use of complementary medicine as well as satisfaction with the complementary treatments among colorectal cancer patients should be assessed in the future NHIS to enhance comprehensiveness of the analysis. As the NHIS depends on retrospective self-reported data, the patients may possibly have recall bias regarding their complementary medicine use.

\section{Conclusion}

In this study, over one fourth of the US colorectal cancer patients had consumed complementary medicine. Among different types of complementary medicine, mind-body medicine was found to be the most commonly used. With evidence supporting the effectiveness and safety of mind-body medicine use among colorectal cancer patients, promoting the use of evidence-based mind-body medicine for colorectal cancer management could be considered.

Authors' contributions Charlene HL Wong: conceptualization, writing original draft.

Tobias Sundberg: conceptualization, writing original draft.

Vincent CH Chung: conceptualization, writing original draft.

Petra Voiss: conceptualization, methodology, writing, review, and editing.

Holger Cramer: conceptualization, data curation, formal analysis, methodology, visualization, writing original draft.

Funding information Open Access funding provided by Projekt DEAL. HC was supported by the Erich Rothenfußer Stiftung. VP was supported by a grant from the Karl and Veronica Carstens Foundation, Essen, Germany. The funding sources had no influence on the design and conduct of the study; the management, analysis, and interpretation of the 
data; the preparation, review, or approval of the manuscript; or the decision to submit the manuscript for publication.

Data availability The datasets analyzed during the current study are available from the National Center for Health Statistics (NCHS), https://www. cdc.gov/nchs/nhis/nhis_2017_data_release.htm

\section{Compliance with ethical standards}

Conflict of interest The authors declare that they have no conflict of interest.

Ethical approval NCHS Research Ethics Review Board approved NHIS data collection. The protocol was approved by the NCHS ERB on June 12, 2015 (Protocol \#2015-08).

Consent to participate All participants provided informed consent to participate.

Open Access This article is licensed under a Creative Commons Attribution 4.0 International License, which permits use, sharing, adaptation, distribution and reproduction in any medium or format, as long as you give appropriate credit to the original author(s) and the source, provide a link to the Creative Commons licence, and indicate if changes were made. The images or other third party material in this article are included in the article's Creative Commons licence, unless indicated otherwise in a credit line to the material. If material is not included in the article's Creative Commons licence and your intended use is not permitted by statutory regulation or exceeds the permitted use, you will need to obtain permission directly from the copyright holder. To view a copy of this licence, visit http://creativecommons.org/licenses/by/4.0/.

\section{References}

1. International Agency for Research on Cancer, World Health Organization. Colorectal cancer 20182019 [Available from: http://gco.iarc.fr/today/data/factsheets/cancers/10_8_9Colorectum-fact-sheet.pdf]

2. Lee RM, Cardona K, Russell MC (2019) Historical perspective: two decades of progress in treating metastatic colorectal cancer. J Surg Oncol 119:549-563

3. National Cancer Institute. SEER cancer stat facts: colorectal cancer 2019 [Available from: https://seer.cancer.gov/statfacts/html/ colorect.html]

4. Arnold M, Sierra MS, Laversanne M, Soerjomataram I, Jemal A, Bray F (2017) Global patterns and trends in colorectal cancer incidence and mortality. Gut 66:683-691

5. Reynolds A, Mann J, Cummings J, Winter N, Mete E, Te Morenga L (2019) Carbohydrate quality and human health: a series of systematic reviews and meta-analyses. Lancet 393:434-445

6. Tahir MZ (2018) Low prevalence of colorectal cancer in South Asians than White population in UK: probable factors. J Coll Phys Surg 28:631-635

7. Brenner H, Kloor M, Pox CP (2014) Colorectal cancer. Lancet 383 : 1490-1502

8. Kuipers EJ, Grady WM, Lieberman D, Seufferlein T, Sung JJ, Boelens PG, van de Velde CJH, Watanabe T (2015) Colorectal cancer. Nat Rev Dis Primers 1:15065

9. National Cancer Institute. Colon cancer treatment. PdqCancerInfoSummary 2019 [Available from: https://www. cancer.gov/types/colorectal/hp/colon-treatment-pdq]
10. National Cancer Institute. Rectal cancer treatment. PdqCancerInfoSummary 2019 [Available from: https://www. cancer.gov/types/colorectal/hp/rectal-treatment-pdq]

11. Jansen L, Koch L, Brenner H, Arndt V (2010) Quality of life among long-term $(>/=5$ years $)$ colorectal cancer survivors-systematic review. Eur J Cancer 46:2879-2888

12. Eyl RE, Xie K, Koch-Gallenkamp L, Brenner H, Arndt V (2018) Quality of life and physical activity in long-term $(>/=5$ years postdiagnosis) colorectal cancer survivors - systematic review. Health Qual Life Outcomes 16:112

13. Sewitch MJ, Rajput Y (2010) A literature review of complementary and alternative medicine use by colorectal cancer patients. Complement Ther Clin Pract 16:52-56

14. John GM, Hershman DL, Falci L, Shi Z, Tsai WY, Greenlee H (2016) Complementary and alternative medicine use among US cancer survivors. J Cancer Surviv 10:850-864

15. National Center for Health Statistics. National Health Interview Survey 2019 [Available from: https://www.cdc.gov/nchs/nhis/ about_nhis.htm]

16. Centers for Disease Control and Prevention. National Health Interview Survey. Survey description 2018 [Available from: ftp:// ftp.cdc.gov/pub/Health_Statistics/NCHS/Dataset_Documentation/ NHIS/2017/srvydesc.pdf]

17. Ranganathan P, Pramesh CS, Aggarwal R (2017) Common pitfalls in statistical analysis: logistic regression. Perspect Clin Res 8:148151

18. Tough SC, Johnston DW, Verhoef MJ, Arthur K, Bryant H (2002) Complementary and alternative medicine use among colorectal cancer patients in Alberta, Canada. Altern Ther Health Med 8:54$6,58-60,62-4$

19. Molassiotis A, Fernandez-Ortega P, Pud D, Ozden G, Platin N, Hummerston S, Scott JA, Panteli V, Gudmundsdottir G, Selvekerova S, Patiraki E, Kearney N (2005) Complementary and alternative medicine use in colorectal cancer patients in seven European countries. Complement Ther Med 13:251-257

20. Dean-Clower E, Doherty-Gilman AM, Keshaviah A, Baker F, Kaw C, Lu W et al (2010) Acupuncture as palliative therapy for physical symptoms and quality of life for advanced cancer patients. Integr Cancer Ther 9:158-167

21. Buckner CA, Lafrenie RM, Denommee JA, Caswell JM, Want DA (2018) Complementary and alternative medicine use in patients before and after a cancer diagnosis. Curr Oncol 25:e275-e281

22. Fouladbakhsh JM, Stommel M (2010) Gender, symptom experience, and use of complementary and alternative medicine practices among cancer survivors in the U.S. cancer population. Oncol Nurs Forum 37:E7-e15

23. Fouladbakhsh JM, Stommel M, Given BA, Given CW (2005) Predictors of use of complementary and alternative therapies among patients with cancer. Oncol Nurs Forum 32:1115-1122

24. Stein KD, Kaw C, Crammer C, Gansler T (2009) The role of psychological functioning in the use of complementary and alternative methods among disease-free colorectal cancer survivors: a report from the American cancer society's studies of cancer survivors. Cancer 115:4397-4408

25. Archer S, Buxton S, Sheffield D (2015) The effect of creative psychological interventions on psychological outcomes for adult cancer patients: a systematic review of randomised controlled trials. Psychooncology 24:1-10

26. Verhoef MJ, Balneaves LG, Boon HS, Vroegindewey A (2005) Reasons for and characteristics associated with complementary and alternative medicine use among adult cancer patients: a systematic review. Integr Cancer Ther 4:274-286

27. Hietala $M$, Henningson M, Ingvar C, Jonsson PE, Rose C, Jernstrom H (2011) Natural remedy use in a prospective cohort of breast cancer patients in southern Sweden. Acta Oncol 50:134-143 
28. Posadzki P, Watson L, Ernst E (2013) Herb-drug interactions: an overview of systematic reviews. Br J Clin Pharmacol 75:603-618

29. Davis EL, Oh B, Butow PN, Mullan BA, Clarke S (2012) Cancer patient disclosure and patient-doctor communication of complementary and alternative medicine use: a systematic review. Oncologist 17:1475-1481

30. Nissen N, Schunder-Tatzber S, Weidenhammer W, Johannessen H (2012) What attitudes and needs do citizens in Europe have in relation to complementary and alternative medicine? Forsch Komplementmed 19(Suppl 2):9-17

31. Nissen N, Weidenhammer W, Schunder-Tatzber S, Johannessen H (2013) Public health ethics fo complementary and alternative medicine. Eur J Integr Med 5:62-67

32. Nissen N, Lunde A, Pedersen CG, Johannessen H (2014) The use of complementary and alternative medicine after the completion of hospital treatment for colorectal cancer: findings from a questionnaire study in Denmark. BMC Complement Altern Med 14:388

33. Sanford NN, Sher DJ, Ahn C, Aizer AA, Mahal BA (2019) Prevalence and nondisclosure of complementary and alternative medicine use in patients with cancer and cancer survivors in the United States. JAMA Oncol 5:735-737

34. Mayden KD (2012) Mind-body therapies: evidence and implications in advanced oncology practice. J Adv Pract Oncol 3:357-373

35. Atreya CE, Kubo A, Borno HT, Rosenthal B, Campanella M, Rettger JP, Joseph G, Allen IE, Venook AP, Altschuler A, Dhruva A (2018) Being present: a single-arm feasibility study of audiobased mindfulness meditation for colorectal cancer patients and caregivers. PLoS One 13:e0199423
36. Kim KJ, Na YK, Hong HS (2016) Effects of progressive muscle relaxation therapy in colorectal cancer patients. West J Nurs Res 38: 959-973

37. Lu Y, Qu HQ, Chen FY, Li XT, Cai L, Chen S, Sun YY (2019) Effect of Baduanjin qigong exercise on cancer-related fatigue in patients with colorectal cancer undergoing chemotherapy: a randomized controlled trial. Oncol Res Treat 42:431-439

38. Cramer H, Pokhrel B, Fester C, Meier B, Gass F, Lauche R, Eggleston B, Walz M, Michalsen A, Kunz R, Dobos G, Langhorst J (2016) A randomized controlled bicenter trial of yoga for patients with colorectal cancer. Psychooncology 25:412-420

39. Oh B, Butow PN, Mullan BA, Clarke SJ, Beale PJ, Pavlakis N, Lee MS, Rosenthal DS, Larkey L, Vardy J (2012) Effect of medical qigong on cognitive function, quality of life, and a biomarker of inflammation in cancer patients: a randomized controlled trial. Support Care Cancer 20:1235-1242

40. Cramer H, Quinker D, Schumann D, Wardle J, Dobos G, Lauche R (2019) Adverse effects of yoga: a national cross-sectional survey. BMC Complement Altern Med 19:190

41. The ASCO Post. The role of meditation in cancer care 2017 [Available from: https://www.ascopost.com/issues/may-25-2017/ the-role-of-meditation-in-cancer-care/]

Publisher's note Springer Nature remains neutral with regard to jurisdictional claims in published maps and institutional affiliations. 